\title{
Metamaterials for electromagnetic and thermal waves
}

\author{
Erin Donnelly ${ }^{1}$, Antoine Durant ${ }^{1,2}$, Célia Lacoste $^{1,3}$, Luigi La Spada ${ }^{1}$ \\ ${ }^{1}$ School of Engineering and the Built Environment, Edinburgh Napier University, Edinburgh, United Kingdom \\ ${ }^{2}$ IUT1, Université Grenoble Alpes, Grenoble, France \\ ${ }^{3}$ INP-ENSEEIHT, University of Toulouse, Toulouse, France \\ 1.1aspada@napier.ac.uk
}

\begin{abstract}
In the last decade, electromagnetic metamaterials, thanks to their exotic properties and the possibility to control electromagnetic waves at will, become crucial building blocks to develop new and advanced technologies in several practical applications. Even though until now the metamaterial concept has been mostly associated with electromagnetism and optics; the same concepts can be also applied to other wave phenomena, such as thermodynamics. For this reason, here the aim is to realize a multi-functional metamaterial able to control and manipulate simultaneously both electromagnetic and thermal waves. The structure is, first, designed and fabricated by using additive manufacturing techniques. Then, it is tested for the following industrial applications: sensing and medical diagnostics (optical and thermal imaging), military/safety (electromagnetic and thermal guiding structures) and automotive (electrical vehicles battery electric and thermal management). Experimental results reveal that such multifunctional metamaterial can fully manipulate and process both electromagnetic and thermal waves at will. The proposed structure appears to be highly versatile and scalable, with great potential to be used also for other wave phenomena such as mechanics, acoustics and hydrodynamics.
\end{abstract}

Index Terms - Electormagnetics, wave propagation, thermodynamics, imaging, guiding structures, thermal management.

\section{INTRODUCTION}

Electromagnetic metamaterials, and their bi-dimensional counterpart, metasurfaces, have become well-established technologies in electrical/electronic engineering, chemistry, biology and medicine [1]. The main reason for this interest is that these engineered materials display novel and enhanced properties compared to traditional ones, paving the way for the development of new technological applications. For this reason, in the last decade, theoretical models, design and characterization tools, last but not least fabrication techniques have been developed for such materials: their peculiar electromagnetic features are now extensively used in several industrial applications, such as telecommunication, automotive, computer science and sensing/medical diagnostics [2].

Until now, metamaterials have been mainly associated with electromagnetism and optics [3], and only recently similar concepts have been also applied to other wave phenomena, i.e. thermodynamics [4]. Such works are mostly theoretical [5], and experiments are still in their infancy [6].

The possibility of using a single metamaterial to control and manipulate both waves at will (electromagnetic and heat) is of great interest in any industrial applications. Moreover, unsolved issues are still present: significant presence of losses, narrow bandwidth, material properties difficult to achieve, complexity in both design and manufacturing. Therefore, in this paper, we model, design and fabricate a multi-functional metamaterial able to control amplitude and phase of both electromagnetic and thermal wave.

The paper is organized as follows: $(i)$ in the modeling section, by using field theory, we evaluate the metamaterial fundamental parameters: electric permittivity $\varepsilon$ and thermal conductibility, in order to describe the wave-structure interaction for both electromagnetic and heat wave phenomena, respectively; (ii) in the design section, by using circuit theory, we relate the metamaterial parameters to the structure geometry and dimensions, in order to realize the structure as a function of the requested application; (iii) both planar and curvilinear geometries are realized by using Additive Manufacturing techniques; and (iv) tested for the following practical applications: imaging, guiding structures and battery voltage/thermal management.

Experimental results demonstrate the ability of such metamaterial to fully control amplitude and phase of both electromagnetic and heat wave phenomena. This paves the way for innovative harvesting technology to power plants and factories.

\section{MATHERIALS AND METHODS}

Fig.1(a) shows the geometry of a metamaterial formed by metallic (dielectric) inclusion (Fig.1(b)) with thickness $t_{i}$, relative permittivity $\varepsilon_{\mathrm{i}}$ (magnetic permeability of free space $\mu_{\text {slab }}=\mu_{0}$ ) and thermal conducibility $k_{\mathrm{i}}$. With this assumption, the entire structure will have a non-homogeneous permittivity $\varepsilon(\mathbf{r})$, whose value will depend on the vector position $\mathbf{r}$. The surrounding material is considered homogeneous, namely air (permittivity $\varepsilon_{0}$ and thermal conducibility $k_{0}$ ), isotropic and non-absorbing media.

\section{A. Metamaterial modeling: from waves to fields}

In this section, by exploiting field theory and wave equations [7], we will obtain the mathematical description of the interaction between the propagating wave and the metamaterial structure. In other words, we relate the wave components with the structure field components (electric $\mathbf{E}$, temperature $\mathbf{T}$ ) and parameters (electric permittivity $\varepsilon$, thermal conductivity $k$ ). It is known that wave equation in homogeneous materials is easily solvable, and its solutions 
are straightforward for almost all the geometries we can envision [8]. On the other hand, in non-homogeneous media such equation became more complex, and it should satisfy the following differential equation:

$$
\nabla^{2} \mathbf{P}+\nabla[\mathbf{P} \cdot \nabla \log \mathcal{E}(\mathbf{r})]+\omega^{2} \gamma(\mathbf{r}) \mathbf{P}=0
$$

Where the vector $\mathbf{P}$ is the electric $\mathbf{E}$ or the heat conduction T wave component; $\gamma(\mathbf{r})$ the non-homogeneous wave vector for the electromagnetic $k(\mathbf{r})$ or thermal $B(\mathbf{r})$ phenomena, respectively. Similar considerations can be done for the dual vector field components: magnetic $\mathbf{H}$ and convection $\mathbf{C}$.

Solutions of (1) are the field components within the metamaterial. They can take different expressions according to: geometry, boundary conditions and materials properties. Knowing both the electromagnetic and thermal configuration of the structure, its equivalent field impedance can be easily evaluated as:

$$
\begin{aligned}
& Z_{e m}(\mathbf{r})=\frac{\mathbf{E}(\mathbf{r})}{\mathbf{H}(\mathbf{r})} \\
& \mathbf{Z}_{t h}(\mathbf{r})=\frac{\mathbf{T}(\mathbf{r})}{\mathbf{C}(\mathbf{r})}
\end{aligned}
$$

For the electromagnetic case it is the ratio electric $\mathbf{E}$ on magnetic $\mathbf{H}$ field components. For the thermal case, instead is the ratio heat conduction $\mathbf{T}$ and convection $\mathbf{C}$ fields.

From electromagnetic theory, it is known that when a wave is impinging on metallic (dielectric) material, electric E (magnetic H) field components excite electric (magnetic) density currents $\mathbf{J}$. This is analogous in the thermal domain where the difference of temperature $\mathbf{T}$ will rise both conduction and convection density currents $\boldsymbol{Q}$ across the material with similar behavior of the electromagnetic domain. We can associate field and current densities vectors to their scalar counterparts for both the electromagnetic and thermal wave phenomena as summarized in Table 1.

\begin{tabular}{|c|c|c|}
\hline & Vectors & Scalars \\
\hline \multirow{5}{*}{$\begin{array}{c}\mathbf{E M} \\
{[9]}\end{array}$} & $\begin{array}{c}\text { Electric field } \\
\mathbf{E} \\
\end{array}$ & $\begin{array}{c}\text { Voltage } \\
\Delta v\end{array}$ \\
\hline & $\begin{array}{l}\text { Magnetic field } \\
\text { H }\end{array}$ & $\begin{array}{c}\text { Current } \\
i\end{array}$ \\
\hline & $\begin{array}{c}\text { Electric current density } \\
\mathbf{J}_{\mathrm{e}}=\boldsymbol{\sigma} \mathbf{E}\end{array}$ & $\begin{array}{l}\text { Electric conduction current } \\
i_{e}=v / R\end{array}$ \\
\hline & $\begin{array}{c}\text { Electric displacement current } \\
\mathbf{J}_{\mathbf{d}}=\mathbf{j} \omega \boldsymbol{\varepsilon} \mathbf{E}\end{array}$ & $\begin{array}{c}\text { Current through a Capacitor } \\
i_{c}=j \omega \mathrm{C} v\end{array}$ \\
\hline & $\begin{array}{l}\text { Magnetic displacement current } \\
\mathbf{J}_{\mathbf{m}}=\mathrm{j} \omega \mu \mathbf{H}\end{array}$ & $\begin{array}{c}\text { Voltage across an inductor } \\
v_{i}=\mathrm{j} \omega \mathrm{L} i\end{array}$ \\
\hline \multirow{5}{*}{$\begin{array}{c}\text { Thermal } \\
{[10]}\end{array}$} & $\begin{array}{c}\text { Conduction Temperature } \\
\mathbf{T} \\
\end{array}$ & $\begin{array}{c}\text { Conduction Potential } \\
\Delta \mathrm{T} \\
\end{array}$ \\
\hline & $\begin{array}{c}\text { Convection } \\
\mathbf{C} \\
\end{array}$ & $\begin{array}{c}\text { Convective Current } \\
i_{q} \\
\end{array}$ \\
\hline & $\begin{array}{c}\text { Conduction flux density } \\
\mathbf{Q}_{\text {cond }}\end{array}$ & $\begin{array}{c}\text { Conduction transfer rate } \\
i_{q} \text { cond } \\
=\Delta \mathrm{T} / \mathrm{R}_{\text {cond }}\end{array}$ \\
\hline & $\begin{array}{c}\text { Forced Convection density } \\
\text { Qforced_conv }^{\text {forced }}\end{array}$ & $\begin{array}{c}\text { Forced convection transfer rate } \\
i_{\text {forced conv }}=\Delta \mathrm{T} / \mathrm{C}_{\text {thermal }}\end{array}$ \\
\hline & $\begin{array}{c}\text { Free Convection density } \\
\mathbf{Q}_{\text {free_conv }}\end{array}$ & $\begin{array}{c}\text { Free convection transfer rate } \\
\Delta \mathrm{T}=\mathrm{L}_{\text {conv }} i_{\text {free }} \text { conv }\end{array}$ \\
\hline
\end{tabular}

TABLE I. TRANSFORMATION TABLE FROM FIELD VECTORS TO CIRCUIT SCALAR

\section{B. Metamaterial design: from fields to circuits}

In this paragraph by using circuit theory [11], we will link the structure electromagnetic and thermal phenomena (fields and currents) with its inclusion geometry and dimensions. By using a similar analogy developed in [12], we represent the structure material properties (permittivity $\varepsilon$, thermal conductivity $k$ ) with its equivalent electrical and thermal circuit lumped elements (Resistance R, Capacitance $\mathrm{C}$ and Inductance L), characterizing its lumped circuit impedance $Z$. From [12] it is known that the equivalent electrical circuit model for a generic shape particle is either a capacitor or a inductor, depending on the real part of its electric permittivity, being positive, $\operatorname{Re}\{\varepsilon\}>0$, or negative, $\operatorname{Re}\{\varepsilon\}<0$, respectively. In addition, the imaginary part of the material permittivity $\operatorname{Im}\{\varepsilon\} \neq 0$ provides an equivalent resistor. For analogy, we consider the sign of the real part of thermal parameter $k$ in eq. (3); therefore, we can write the equivalences summarized in Table 2. The equivalent electrical and thermal circuit model for the single inclusion is reported in Fig.1(c), and Fig.1(d), respectively.

TABLE II. EQUIVALENCES FOR ELECTRICAL AND THERMAL CIRCUIT ELEMENTS

\begin{tabular}{|c|c|c|}
\hline Element & Electrical Circuit [11] & Thermal Circuit [10] \\
\hline Resistance & $R_{\text {ele }}=\rho l / A$ & $R_{\text {cond }}=L /(\mathrm{kA}), R_{\text {conv }}=1 / \mathrm{hA}$ \\
\hline Capacitance & $C_{e l e}=\varepsilon A / d$ & $C_{t h}=m_{h} c_{p h}$ \\
\hline Inductance & $L_{e l e}=\left(N^{2} \mu A\right) / d$ & $L_{t h}=L_{e l e} / \alpha^{2} T[13]$ \\
\hline
\end{tabular}

Once we determine the relation between the structure electromagnetic/thermal properties and its physical dimensions, in the next paragraph we will choose and manufacture the metamaterial accordingly to the target and application required. (a)

(c)
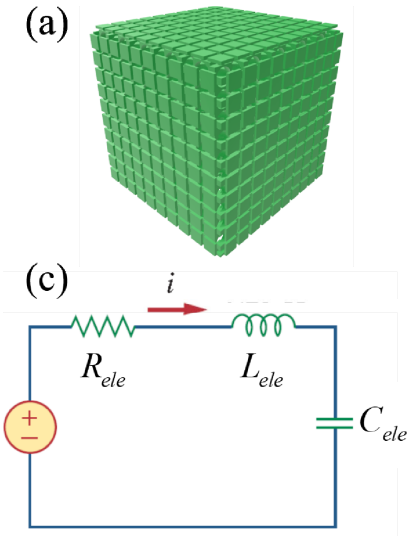

(d)
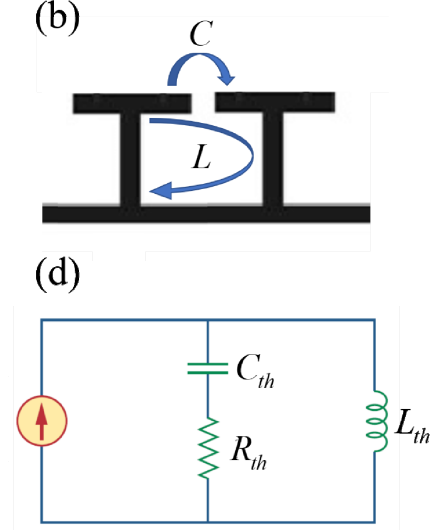

Fig. 1. (a) Generic metamaterial conmposed by mushroom-tipe inclusions; (b) Side view of the unit-cell showing electromagnetic/thermal field lines (capacitance $C$ ) and vortexes (inductance $L$ ); Equivalent circuit model for (c) electromagnetic and (d) thermal phenomena.

\section{Metamaterial manufacturing}

The Additive manufacturing (AM) technique consists in a multi-step process, involving three stages:

(i) Particulate Filler Preparation (Fig.2(a)): each layer is manufactured by dispersing filler materials (ceramic-based 
materials) into a resin matrix. A dedicated cure regime is needed to generate the powders with particle size distributions of micro- and nano- meter size. As dictated by the modeling/design approach, both permittivity $\varepsilon$ and thermal conductivity $k$ have been controlled through the simultaneously tailoring of the following: dispersion of the fillers, particle size distribution, particulate shape, compatibility with the resin system, curing process, and volume fraction.

(ii) Composite Production (Fig.2(b)): In order to achieve the desired electromagnetic and thermal properties in every layer of the composite structure, the filler and the matrix were pre-processed separately in a vacuum system prior to forming the mixture. Subsequently, the filler was added to the resin system under vacuum to reduce the void content. This was necessary since the dispersed powder should remain in a stable suspension during the cure cycle and to not settle out. To control the thickness of each layer, a blade was used to enable micro-meters adjustments.

(iii) Multi-cast sequential layer assembly (Fig.2(c)): To produce the final structure the optimized mixture has been casted sequentially onto the target volume/surface. Once cured, the top surface of the structure was machined to match the desired profile. In this case, for each casting mixtures we needed to control simultaneously its permittivity, thermal conductivity and mixture rheology. (a)

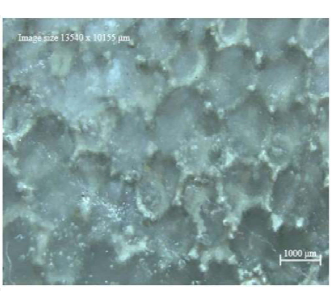

(c)

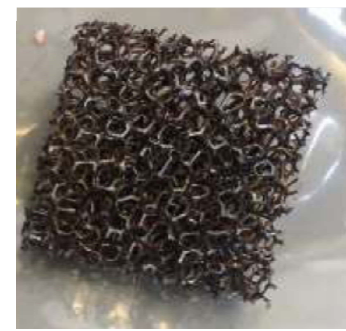

(b)
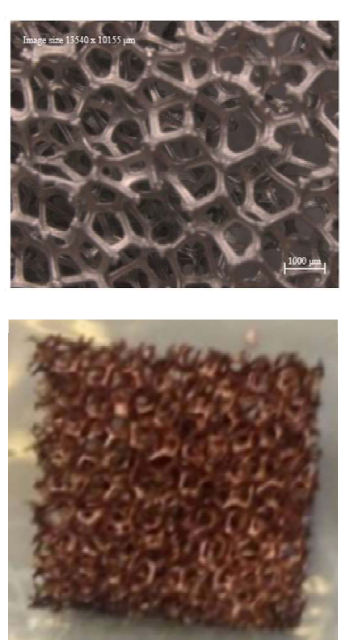

Fig. 2. Manufacturing steps: (a) Particulate Filler Preparation and (b) Composite Production; (c) Manufactured samples: copper-based (left); copper/nickel-based (right)

\section{RESULTS AND DISCUSSION}

Any wave phenomena in presence of boundaries can be always modelled as follows: $(i)$ a transmitted wave thorough the discontinuity into air (radiated waves); (ii) a reflected wave travelling within the structure (wave-guide modes); (iii) an evanescent wave travelling parallel to the interface (surface waves). The different form that the wave can assume is affected by the boundary conditions existing at the interface, mainly: electromagnetic/thermal properties of the materials involved, and their geometries/shapes. By using the approach proposed in the previous paragraph, we will manipulate both electromagnetic/thermal parameters and the material geometry/shape to create a metamaterial that can be used for the following practical applications: optical/thermal imaging, electromagnetic/thermal guiding structures and electric/thermal power management.

(a)
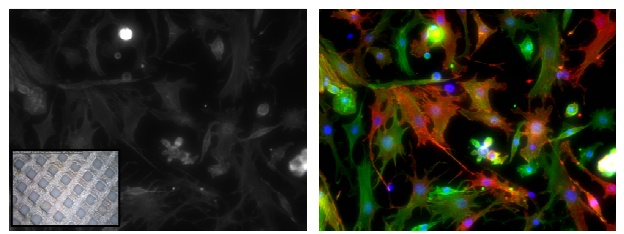

(b)
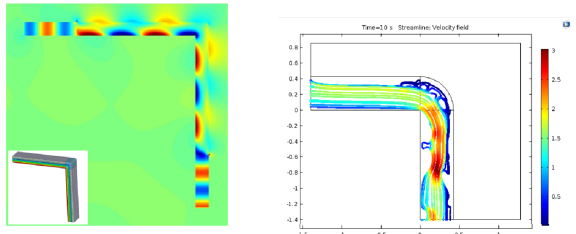

(c)
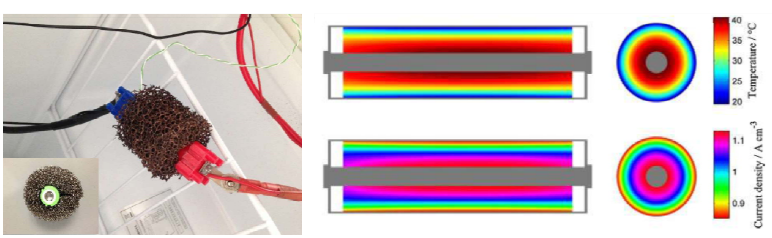

Fig. 3. Condrocytes from the articular cartilage of knee joint: (a) optical (left) and thermal (right) imaging technique; (b) Electromagnetic (left) and thermal (right) waveguide around 90 degrees corner; (c) battery voltage and thermal management: experimental setup (left) and results (right).

\section{A. Imagning for medical diagnostics}

Radiated waves find their applications in different industrial sectors, such as [14]: modulators, camouflage, photovoltaics, wireless devices and absorbers. This concept is of great interest in medical diagnostics. The primary aim of a sensor is to produce an output (signal or image), related to the type/concentration of the sample under study [15]. Diseases in biological samples typically induce structural, biochemical and mechanical variations [16], implying significant modifications in both electromagnetic and thermal properties [17], namely: refractive index $n$ and thermal conductibility $k$. The main aim of the sensor is to reveal such differences, by correlating the sample properties to the output signal characteristics in terms of amplitude (image/temperature intensity) and phase (resolution/mass transfer). By using our approach, we combined both concepts of optical metasurfaces [18] and thermal shifters [19] to realize an optical imaging structure; in order to detect primary culture of condrocytes, collected from the articular cartilage of knee joint, hip and elbow of mice 10/12 days old [20]. The unit-cell of the planar metasurface, shown in the inset of Fig3(a), has been designed by using the following circuit impedance:

$$
\mathbf{Z}_{x, y, z}=\mathbf{R}_{\text {tot }}+\frac{1-\omega^{2} \mathbf{L}_{\text {tot }} \mathbf{C}_{\text {tot }}}{j \omega \mathbf{C}_{\text {tot }}}
$$


Subsequently, manufactured by using square patches with the following sizes: side length $l=\lambda_{0} / 15$, strip width $w=$ $\lambda_{0} / 75$ and thickness $t=\lambda_{0} / 30$; printed on a grounded dielectric slab (with uniform permittivity value $\varepsilon_{\mathrm{r}}=15$ and thickness $d=\lambda_{0} / 10$ ), being $\lambda_{0}$ the free-space wavelength. Experimental results are reported in Fig3(a) for both electromagnetic and thermal imaging of the sample under test. The structure has the capability to detect biological cell interactions in real time with subwavelength resolution. This is extremely useful for observing a whole living cell and the related cellular processes, such as how proteins and fats move in and out of cells. Cellular processes involving mass transfer (i.e. the dynamic motions of proteins) will be observable to target a specific type of human red blood cell protein that forms part of a network structure in the cell's inner membrane. In this way an infection can be detected, and its progress with its several distinct developmental stages can be easily followed.

\section{B. Guiding structures for electromagnetic and thermal waves}

Waveguides and optical fibres are crucial components in integrated circuits and communication technologies [21]. Structures encountered in practice often exhibit sharp wedges, vertices, or corners. The presence of any abrupt discontinuities typically leads to pronounced electromagnetic and thermal losses and consequently high attenuation of the propagating wave. The possibility to guide both waves around corners and/or discontinuities, without affecting their amplitude and phase, results of great importance in such systems.

We combined both concepts of curvilinear metamaterials [22] and thermal exchanger [23]; we realize a curvilinear structure to bend both waves around discontinuities (sharp 90-degrees corners). From the planar impedance profile described in (3), by using the following Elliptical Cylindrical Coordinates $(\mu, v, z)$ transformation:

$$
\mathbf{Z}_{\boldsymbol{\mu , v , \mathbf { z }}}=\left(\begin{array}{ccc}
0 & -1 & a \cosh ^{2} \mu \\
0 & 1 & a^{2} \cos ^{2} v \\
1 & 0 & 1
\end{array}\right) \mathbf{Z}_{x, y, z}
$$

We designed and manufactured the curvilinear nonhomogenous metamaterial, accordingly. Experimental results are shown in Fig3(b). The structure is made of 7 different layers with an electric permittivity range of $2.3-$ 35. Each layer has a thickness of ...

From the electromagnetic point of view, the wave is trapped inside the slab at constant velocity forming a standing wave. At the corner/discontinuity the wave drastically modifies direction, velocity, and pattern due to the different incident angle. For the wave pattern across the entire slab to remain the same along all the curvature, all multiple reflected waves must add in phase. The permittivity values, used here, determine a special boundary condition named Perfect-ElectroMagnetic Conductor (PEMC) [24], where the PEC is sustained by the high impedance layer $\left(\varepsilon_{\mathrm{r}}=35\right)$ and the PMC is developed by the low impedance one $\left(\varepsilon_{\mathrm{r}}=2.3\right)$.
The electromagnetic energy transported across the crosssection must remain constant, therefore the velocities of the wave components should change locally (faster externally where the curvature is larger, slower internally where the curvature is smaller): in this way the energy is conserved both in amplitude and in phase.

From the fluid-dynamics (thermal) point of view we can see the curvilinear channel, around the sharp corner, acting as a narrow pipe connecting two straight conduits. To go through this tight channel, fluids should increase its velocity profile to maintain constant the flux, at the bottom section with lower temperature the thermal impedance is low, and at the top one with higher temperature the thermal impedance is high. Like in the electromagnetic case, for energy and mass conservation, the velocity profile tends to be $0(v=0)$ and high $(v=\infty)$, at the bottom and top sections, respectively.

Manipulating guided-waves by using metamaterials is crucial to develop: $(i)$ devices for communications, where the aim is to carry stronger signals with higher bandwidth and transmission rates by minimizing both electromagnetic losses and reducing dissipation problems; and (ii) military applications, where the aim is both the electromagnetic and thermal cloaking/camouflage of military vehicles.

\section{Surface-waves and electrical vehicles applications}

Surface waves exists at the interface between media with different electromagnetic/thermal properties such as: stratified planar structures or even more complicate curved surfaces [25]. Controlling them in complex geometrical environments is of great importance in the automotive industrial sectors [26]. Electrical vehicles batteries produce heat during their use and are exposed to a range of ambient temperatures: this strongly affect the engine efficiency. The role of a battery thermal management systems (BTMS) is to keep it within acceptable thermal operating conditions, without affecting the electrical operation. A perfect BTMS design will account for all the dynamic conditions through intelligent control of both the electromagnetic aspect (voltage/current supply) and thermal phenomenon (cooling/heating temperature). To this regard, here, we combined both concepts of electromagnetic Transformation Optics (TO) [27] and Transformation Thermodynamics [28] to realize a cylindrical metamaterial. Starting from the planar impedance expression in (3), and apply the following cylindrical $(r, \phi, z)$ distribution to obtain the metamaterial dimensions:

$$
\mathbf{Z}_{r, \phi, z}=\left(\begin{array}{ccc}
0 & -1 / r^{2} & -1 \\
0 & 1 & 0 \\
1 & 0 & 1
\end{array}\right) \mathbf{Z}_{x, y, z}
$$

By using the manufacturing technique previously mentioned, both nickel, copper and copper/nickel -based samples have been fabricated.

To test our electromagnetic-thermal theory in real-life laboratory conditions, Single Panasonic NCR18650B battery cells were discharged from a state of full capacity. 
The performance results of the realized structure are depicted in Fig3(c).

During discharge, temperature within a battery cell increases at a rate proportional to the load applied. Similarly, temperature rise, and power drawn from a battery have a proportional relationship.

The metamaterial cover copper-based increased surface heat transfer rates most effectively for a better cooling effect. When comparing a steel plate's temperature rise over the first 500 seconds of heat application, the copper samples increased temperature rise rate by a minimum of $28.6^{\circ} \mathrm{C} / \mathrm{s}$ per $\mathrm{kg}$. This was over $11^{\circ} \mathrm{C} / \mathrm{s}$ per $\mathrm{kg}$ higher than the effect observed in copper/nickel-based foam samples.

The metamaterial cover can be used in a larger scale to enhance the performance of Lithium-Ion battery cells in electric vehicles, avoiding traditional issues like Goldilocks effect, permanent and extreme cells damage and to slow its degradation. Therefore, the battery can work at its best without becoming too cold or too hot, being unaffected by surface and environmental temperature conditions.

\section{CONCLUSIONS}

A multi-functional metamaterial able to control both electromagnetic and heat waves have been designed, manufactured and practically tested for imaging, cloaking/camouflage and electrical vehicles applications. The design method, here proposed, permits to obtain full control of the metamaterial electromagnetic and thermal response; most importantly by using this approach, we can manipulate at will its features accordingly to the required application.

The additive manufacturing technique here proposed, brings two main advantages: (i) full control of both size and shape during the fabrication stage; (ii) high reproducibility (different frequency ranges) and scalability (different shape and geometry used).

Such characteristics have been also confirmed by the experimental results carried out on the three different applications. The metamaterial showed high performances in terms of both electromagnetic and thermal wave control.

The multi-factional metamaterial concept, with the appropriate corrections and analogies, can be extended to other physical phenomena based on wave/field equations such as acoustics, fluid dynamics and classical mechanics.

\section{REFERENCES}

[1] N. Engheta and R. W. Ziolkowski, Metamaterials: Physics and Engineering Explorations, John Wiley \& Sons, 2006.

[2] F. Capolino, Applications of Metamaterials, CRC Press, Taylor \& Francis Group, 2017

[3] M. Kadic, et al., "3D metamaterials," Nature Reviews Physics, Volume 1, pages 198-210, 2019.

[4] S. R Sklan and B. Li, "Thermal metamaterials: functions and prospects," National Science Review, Volume 5(2), Pages 138-141, 2018

[5] T. Han, et al., "Full Control and Manipulation of Heat Signatures: Cloaking, Camouflage and Thermal Metamaterials," Advanced Materials, vol. 26 (11), 1731-1734, 2014.
[6] F. Chen and D. Y. Lei, "Experimental Realization of Extreme Heat Flux Concentration with Easy-to-Make Thermal Metamaterials," Scientific Reports, vol. 5, 11552, 2015

[7] P. Moon and D. E. Spencer, Field Theory Handbook, Berlin, Germany, Springer-Verlag, 1963

[8] L. P. Eisenhart, Separable systems in euclidean 3-space. Phys. Rev. 1934, 45, 427.

[9] N. Engheta, "Circuits with Light at Nanoscales: Optical Nanocircuits Inspired by Metamaterials," Science 317, 1698-1702, 2007

[10] Y. A. Cengel, Afshin J.Ghajar, Heat and mass transfer: Fundamentals and Applications, $5^{\text {th }}$ Edition, Mc Graw Hill Education, New York, 2015

[11] C. Alexander, M. Sadiku, Fundamentals of electric circuits, $5^{\text {th }}$ Edition, Mc Graw Hill Education, New York, 2013

[12] N. Engheta, A. Salandrino, and A. Alù, Phys. Rev. Lett. 95, 095504 2005.

[13] A. Schilling, X. Zhang and O. Bossen, "Heat flowing from cold to hot without external intervention by using a "thermal inductor"," Science Advances, Vol. 5(4), 9953, 2019.

[14] C. M. Watts, X. Liu and W. J. Padilla, "Metamaterial Electromagnetic Wave Absorbers," Vol. 24 (23), OP98-OP120, 2012.

[15] L La Spada, L Vegni, "Electromagnetic Nanoparticles for Sensing and Medical Diagnostic Applications," Materials, vol. 11(4), 603, 2018.

[16] DM Wiatrek, et al., "Activation of innate immunity by mitochondrial dsRNA in mouse cells lacking p53 protein,” RNA, 25 (6), 713-726, 2019.

[17] Gabriel, S. "The dielectric properties of biological tissues," Phys. Med. Biol., 41, 2271-2293, 1996.

[18] D. Neshev and I. Aharonovich," Optical metasurfaces: new generation building blocks for multi-functional optics," Light: Science \& Applications, vol. 7, 58, 2018.

[19] G. Park, et al., "Tunable Multifunctional Thermal Metamaterials: Manipulation of Local Heat Flux via Assembly of Unit-Cell Thermal Shifters," Scientific Reports, vol. 7, 41000, 2017.

[20] M. E. Candela, et al., "Alpha 5 integrin mediates osteoarthritic changes in mouse knee joints," PloS one, vol. 11 (6), e0156783, 2016.

[21] A. Poddubny, I. Iorsh, P. Belov and Y. Kivshar, "Hyperbolic metamaterials," Nature Photonics, vol. 7, 948-957, 2013

[22] L. La Spada, et al., "Curvilinear MetaSurfaces for Surface Wave Manipulation, Sci. Rep. 9, 3107, 2019.

[23] P. R. Bandarua, et al., "Layered thermal metamaterials for the directing and harvesting of conductive heat," AIP Advances, 5, 053403, 2015.

[24] L. La Spada and L. Vegni, "Near-zero-index wires," Opt. Express 25, 23699-23708, 2017.

[25] J. Polo, T. Mackay, and A. Lakhtakia, Electromagnetic Surface Waves: A Modern Perspective, Elsevier, USA, 2013.

[26] D. Worwooda et al., "A new approach to the internal thermal management of cylindrical battery cells for automotive applications," Journal of Power Sources, vol. 346, 151-166, 2017.

[27] TM McManus, L La Spada, Y Hao, "Isotropic and anisotropic surface wave cloaking techniques," Journal of Optics 18 (4), 044005, 2016.

[28] M. Raza et al., "Transformation thermodynamics and heat cloaking: a review," Journal of Optics, vol. 18(4), 2016. 